\title{
El financiamiento de las revistas médicas
}

\author{
HUMBERTO REYES B.
}

Editor, Revista Médica de Chile.

Declaración de conflictos de intereses: el autor es funcionario de la Sociedad Médica de Santiago, entidad que encargó este estudio pero no participó en su contenido ni en su publicación, lo cual es responsabilidad del autor.

Correspondencia a: Dr. Humberto Reyes B. E-mail. revmedchile@ smschile.cl

\section{Publishing models in medical journals}

Medical journals are published by scientific societies, universities, publishing agencies and other for-profit or non-profit organizations. The traditional way to cover the expenses in printed journals has been a "subscribers pay" model. The rise of electronic versions in the internet, either together with the printed version or replacing it entirely, plus a progressive adherence to an "open access" for electronic versions, has created financial difficulties. Therefore, the "authors pay" model has been added. Both models can be subsidized by commercial or institutional advertising, but still a main source for financing relies either in subscriptions or in authors' payments. A small source of income that helps to cover publishing costs is a "charge for manuscript reception", currently applied by several journals. Those authors whose work has institutional or external support can use their grants to cover any charges, but the situation is more difficult for those who do not have such support. Since 1872, Sociedad Médica de Santiago-Chilean Society of Internal Medicine, owner and publisher of Revista Médica de Chile, has employed the "subscribers pay" model, subsidized by commercial advertising and temporary sponsors (Chilean government and others). The printed journal is reproduced in an open access electronic version, in www.scielo. cl. The increasing cost of both publications systems demands a time for reflection.

(Rev Med Chile 2012; 140: 266-270).

Key words: Access to information; Journalism, medical; Periodicals as topic; Publishing.
$\mathrm{E}$ n el año 2007, durante la presidencia del Dr. Pedro Paulo Marín L., el Directorio de la Sociedad Médica de Santiago-Sociedad Chilena de Medicina Interna, encomendó al Editor de la Revista Médica de Chile un análisis sobre las modalidades de financiamiento de las revistas médicas, en el ámbito internacional. Existía preocupación por un aumento progresivo en el costo de impresión de la Revista Médica de Chile y su distribución a los suscriptores. Se había producido, además, un cambio importante en los criterios de asignación de recursos por el Fondo de Ayuda a las Publicaciones Científicas, de la Comisión Nacional de Investigación Científica y Tecnológica, que repercutiría negativamente en la oportunidad de recibir ayuda de dicho Fondo para afrontar los gastos permanentes de esta revista.

El Editor realizó una búsqueda bibliográfica en Google y en MEDLINE/PubMed, usando como buscadores "Journalism, medical" AND
"Publishing" OR "Publishing models", desde 1990 a 2007, actualizándola posteriormente hasta enero de 2012. Las referencias obtenidas procedieron mayoritariamente de empresas dedicadas a la edición y distribución de revistas científicas y estaban enfocadas a las publicaciones electrónicas y el acceso libre ("open access"), pronosticando que el financiamiento de las revistas (impresas en papel o electrónicas) mediante suscripciones no durará más de 8 a 9 años ${ }^{1-6}$.

Entre las referencias consultadas destacó un documento que contenía descripciones y juicios fundamentados sobre el tema ${ }^{1}$. Este informe, titulado "Scientific Publications: Free for all?", fue difundido en julio de 2004 por el Comité de Ciencia y Tecnología de la "House of Commons" (Parlamento) del Reino Unido. El Comité, con once miembros designados por el Parlamento Británico, contó con la asesoría de 127 expertos ("witnesses"): editores de revistas científicas, represen- 
tantes de sociedades científicas y profesionales, de corporaciones farmacéuticas, de empresas editoras o impresoras de revistas, de entidades tecnológicas dedicadas a las publicaciones digitales, y otros, incluyendo representantes de la comunidad y personas invitadas desde Canadá, Estados Unidos de Norteamérica y países de Europa. El informe ocupó algo más de 110 páginas, identificando, en los párrafos correspondientes, a los participantes y sus respectivas opiniones.

El Editor de la Revista Médica de Chile consideró ese documento como el análisis más completo y balanceado del tema y se basó en sus contenidos para elaborar una síntesis que presentó al Directorio de la Sociedad, en enero de 2008. La situación actual de la Revista lo estimuló a actualizarlo y difundirlo a los lectores.

\section{Antecedentes}

En el mundo existen más de 16.000 revistas que publican artículos de investigación, tecnología y medicina, reproduciendo aproximadamente 1,2 millones de artículos por año. Poco más de la mitad de estas revistas son publicadas por sociedades científicas ("learned societies", sociedades eruditas) o por universidades; las demás son publicadas por entidades comerciales (empresas editoras, como es el caso de The Lancet) o por organizaciones sin fines de lucro, como la Public Library of Science y sus publicaciones electrónicas PLoS.

Las sociedades científicas son organizaciones sin fines de lucro que publican revistas para aumentar su prestigio institucional, facilitar la difusión del conocimiento creado por sus miembros y estimular la cultura científica de sus lectores. Sin embargo, cuando una sociedad científica o una universidad editan una revista para ofrecerla al ámbito internacional de lectores, no condicionan la aceptación de los manuscritos a la membresía de los autores ni tampoco les ofrecen un trato especial en el proceso editorial, el cual se realiza con absoluta independencia técnica.

Desde el punto de vista financiero, la mayoría de las sociedades pretende solamente recuperar el costo de publicación de sus revistas y, si tienen ganancias, las destinan a perfeccionar la revista o a financiar otras actividades institucionales, tales como cursos, conferencias, becas a jóvenes miembros, etc.

\section{Modalidades de financiamiento de las revistas científicas}

Para financiar la publicación de sus revistas, las sociedades científicas y las empresas editoras pueden optar entre dos modalidades: 1) Pagan los suscriptores, o 2) Pagan los autores. Ambas modalidades pueden estar subsidiadas por la captación de recursos con avisos comerciales (ejs: industria farmacéutica y fábricas de equipos médicos), avisos que difunden actividades de otras organizaciones científicas (cursos, congresos), la oferta de cargos o plazas profesionales y otros. En algunos países, el estado asume la publicación de revistas nacionales.

El "pago por los suscriptores" es la modalidad más antigua y sigue siendo la predominante, en particular en las revistas con mayor prestigio y difusión internacional. La suscripción se incluye en las cuotas societarias, o es pagada por bibliotecas, lectores no-miembros de la sociedad, académicos $\mathrm{y}$ docentes universitarios.

Muchas sociedades enfrentan dificultades motivadas por el costo de la impresión y distribución de sus revistas, la mayor complejidad administrativa y de los procesos editoriales, y una disminución del número de suscriptores.

La aparición de versiones electrónicas de las revistas, con acceso libre universal ("open access") podría disminuir las suscripciones a sus versiones impresas en papel. Este cambio no ha perjudicado a revistas de gran prestigio, que imprimen varios miles de ejemplares (Luis Gabriel Cuervo, Editor Clínico de BMJ Publishing Group; Michael Berkwits, Deputy Editor, Annals of Internal Medicine: comunicaciones personales, 4 de agosto de 2004 y 7 de diciembre de 2011, respectivamente), pero sí ha motivado cambios de política en otras ${ }^{7}$. Para las revistas con menos suscriptores, una disminución en su número puede afectar fuertemente las finanzas institucionales.

Entre las revistas que emplean la modalidad de "pago por los suscriptores" algunas aplican a los autores de manuscritos un cobro de "gastos por recepción de manuscritos", que es una forma de evitar tener que aumentar el valor de las cuotas societarias y de las suscripciones a los no-socios. Su monto no pretende cubrir el costo de publicación de la revista.

La mayoría de las revistas impresas en papel cobran a los autores por publicar figuras en colores. Las revistas que se publican sólo en versión electrónica no aplican cargos por figuras en colores. 
En la modalidad de "pago por los autores", el costo de publicación de la revista debe ser asumido por los autores de los manuscritos publicados, incluyendo los gastos del proceso editorial, los de impresión y distribución. En el cálculo del costo de producción de la revista se resta la recaudación por suscripciones, subvenciones estatales, avisos comerciales, etc. El pago no procede necesariamente del bolsillo de los autores, porque suele ser asumido por las instituciones que financiaron la investigación. Sin embargo, crea una diferencia importante entre las revistas que publican artículos de investigación cuyos autores tienen financiamiento institucional y las que difunden experiencias que surgen del trabajo cotidiano de los autores, sin financiamiento institucional.

Esta modalidad ha sido adoptada por una minoría de las revistas médicas (estimada en medios europeos y norteamericanos, en el año 2003, como inferior al 5\%) pero muestra una tendencia a crecer. Está habitualmente ligada al "open access", o acceso gratuito inmediato y universal a una versión electrónica de la revista.

$\mathrm{El}$ "pago por los autores" incluye los gastos del proceso editorial a que se someten todos los manuscritos recibidos, lo que aumenta el cobro para los manuscritos publicados, porque se reparten el costo de la revisión de los manuscritos rechazados. Mientras más alto sea el índice de rechazos en una revista, mayor es el costo para los aceptados: $\mathrm{Na}$ ture, con $90 \%$ de manuscritos rechazados, cobra una de las cifras más altas por página publicada. En algunas revistas esto se aminora cobrando "gastos de recepción" a todos los manuscritos recibidos, lo cual no se devuelve en caso de rechazo.

Las revistas que recurren al "pago por los autores", cobran montos variados, notoriamente mayores cuando la revista tiene acceso libre en la Internet. En Chile, "Biological Research" (órgano oficial de la Sociedad de Biología de Chile) se imprime en papel y, además, se difunde con acceso libre en la página web de SciELO Chile; cobra US\$ 72 por página impresa y US\$ 600 por página con Figuras en colores. En el hemisferio norte, el Open Journal of Internal Medicine, del Scientific Research Publishing (revista con sólo versión electrónica) cobra US $\$ 500$ a los manuscritos hasta 10 páginas de extensión, más US $\$ 50$ por cada página extra ${ }^{8}$. Probablemente en la situación extrema están las revistas electrónicas de la Public Library of Science (PLoS), que cobran US\$ 2.900 por manuscrito, cualquiera sea su extensión?.
La modalidad "pago por los autores" genera problemas que deben ser sopesados al momento de elegirla:

a) El cobro por publicar es resentido por los autores, particularmente por los que no tienen financiamiento institucional, quienes deberán asumirlo personalmente. Si se trata de autores en comienzos de su carrera profesional, disminuye su interés por aquella revista que cobre por publicar. Algunas revistas, particularmente las electrónicas de mayor costo, ofrecen la posibilidad de condonar el pago o disminuir el monto para autores que declaren ser incapaces de pagarlo9. Estas solicitudes deben ser manejadas por una instancia administrativa ajena a los editores de la revista, para evitar que sus decisiones puedan estar sesgadas (o parezcan estarlo) por la capacidad de pago de los autores.

b) Cuando se exige un "pago por recepción del manuscrito", los autores pueden tener dificultades para justificarlo ante sus instituciones si el manuscrito fuera rechazado. Este cobro genera poca resistencia cuando su monto es bajo (menos de US\$100).

c) La motivación de los autores de experiencias clínicas (análisis retrospectivo de series clínicas o presentación de casos novedosos) tiene matices distintos si se la compara con los autores de artículos de investigación propiamente tales. Los investigadores eligen a las revistas con la intención de despertar interés en sus pares (otros investigadores en su tema); ello les interesa más que su "visualización" por un público general de lectores, porque quieren llamar la atención en su tema específico, de modo que su publicación sea citada después por otros. Por lo tanto, la mayoría de los investigadores se preocupan más de quiénes leyeron su trabajo y podrán citarlo, que de cuántas personas lo han leído. Para los autores de manuscritos, el "factor de impacto" de las revistas en que publican tiene gran relevancia, mientras es marginal para los lectores que no desean publicar.

d) En encuestas en Europa, los investigadores se mostraron más conformes con el financiamiento de las revistas por sus suscriptores, mientras los autores que deseaban una mayor diseminación visual de sus manuscritos fueron más receptivos al modelo de publicación pagada por los autores ${ }^{1}$

e) No se debe aplicar cobros "por página" o por 
"gastos de recepción" para aquellos artículos que se generan por invitación de los editores. Pero, en el espectro de variedad de los manuscritos, que va desde los artículos de investigación (en que se justificaría el pago por los autores) hasta las cartas al editor, editoriales y otros manuscritos por invitación (donde no debe haber cobro), puede generarse un "área gris", difícil de manejar, arriesgando dañar el prestigio de la revista y el interés de los autores por publicar en ella.

f) El cambio de modalidad de una revista financiada por suscriptores a una que cobra a los autores crea dificultades iniciales en la captación de manuscritos, por la reacción negativa de los autores que prefieran enviar su manuscrito a una revista que no les cobre. La sociedad o entidad dueña de la revista (que asume la responsabilidad del cobro) debe estar preparada para justificarlo y aplicarlo con transparencia, incluyendo la entrega de facturas o recibos legales para cada recurso recaudado.

g) El cobro a los autores puede afectar la buena disposición de los revisores externos, quienes participan gratuita y generosamente en el proceso de revisión por pares. La mayoría de los revisores son también autores de manuscritos y su voluntad para colaborar como revisores podría resentirse si viven experiencias negativas en su rol como autores.

Una posible ventaja del cobro a los autores, sería que los motive a ser más cuidadosos para redactar lo que mandan a una revista, sobre todo si se les cobra por la sola recepción del manuscrito.

\section{Situación de la Sociedad Médica de Santiago- Sociedad Chilena de Medicina Interna y su publicación principal, la Revista Médica de Chile}

En mayo de 1872, la Sociedad Médica de Santiago acordó "la fundación de un periódico de medicina $i$ ciencias naturales como lo prescriben los estatutos generales" y en julio del mismo año apareció el primer número de la Revista Médica. Desde entonces hasta hoy, la publicación de la Revista se ha financiado con las cuotas de los socios, la inserción de avisos comerciales, la venta de suscripciones a no-socios y bibliotecas y aportes externos especiales.

Esta modalidad de "pago por suscriptores" ha tenido altibajos en la captación de recursos, según los registros históricos recogidos por el Dr. Camilo Larraín en su libro "La Sociedad Médica de Santiago y el Desarrollo Histórico de la Medicina en Chile"10. En el primer medio siglo de la Revista, el problema fundamental para sus editores fue la dificultad para conseguir artículos escritos por médicos del país, provocando retrasos en la aparición de muchos números, por lo que, en su lugar, se publicaron traducciones de artículos seleccionados en otras revistas, principalmente europeas. Circa 1907 el Directorio de la Sociedad "recurrió al arbitrio" de remunerar algunos trabajos de revisión o actualización escritos por médicos chilenos, permitiendo a la Revista difundir un material que -si bien no era original- presentaba a los lectores "un panorama de los conocimientos nuevos y de los adelantos de la ciencia". En la primera mitad del siglo XX, hubo problemas ocasionados por dificultades financieras de la Sociedad Médica de Santiago. En 1957, la Revista aportó a su financiamiento suscripciones y avisos que cubrieron sólo cuatro de los siete millones de pesos que costó su impresión; para cubrir la diferencia se consiguieron aportes de la Universidad de Chile, el Colegio Médico de Chile y otras instituciones. Sin embargo, no hay recuerdo de cobros a los autores para publicar sus manuscritos.

Actualmente, los costos de producción de la Revista Médica de Chile incluyen el uso de oficinas en el edificio de la Sociedad (con sus requerimientos técnicos y de equipos computacionales), honorarios para los editores, sueldos para secretarias y auxiliar, los trámites administrativos que requieren los manuscritos (llamadas telefónicas, correo electrónico), la habilitación de un sistema computacional para la recepción y manejo de los manuscritos, el proceso editorial (con intercambio de comunicaciones entre editores, autores y revisores externos), la impresión de la Revista en papel, su "digitalización" para reproducirse en la página web de SciELO Chile y su distribución (franqueo de correo o reparto a domicilio). Para solventar estos costos, la Sociedad Médica de Santiago ha utilizado parte de las cuotas pagadas por sus socios, más la inserción de avisos comerciales, la venta de suscripciones a no-socios y bibliotecas, y un subsidio de la Comisión Nacional de Investigación Científica y Tecnológica (CONICYT) otorgado desde 1988 hasta 2007, por concurso abierto a las revistas chilenas, de carácter científico o humanís- 
tico, con los recursos del Fondo de Publicación de Revistas Científicas.

De acuerdo a un informe de Ingresos y Egresos disponible a fines de 2007, la publicación y distribución de la Revista Médica de Chile generó en ese año egresos por un monto de $\$ 42.900 .000$; los ingresos fueron $\$ 12.500 .000$ (incluyendo el subsidio de CONICYT), con una diferencia egresos-ingresos de $\$ 30.400 .000$. Para aquellos socios que recibieron la Revista sin pagar cuotas (becados de primer año, socios honorarios, etc.), la Sociedad les regaló una suscripción anual que valía $\$ 28.494$.

A partir de 2008 el subsidio otorgado por el Fondo de Publicación de Revistas Científicas tiene un destino específico que no permite contribuir a financiar la impresión de la Revista, aumentando la brecha egresos-ingresos.

\section{Consideraciones ante un eventual cobro a los autores en la Revista Médica de Chile}

Para analizar la conveniencia de modificar la modalidad de financiamiento de la Revista Médica de Chile, parece razonable considerar lo siguiente:

$1^{\circ}$ Es necesario disponer de un estudio sobre el número de socios de la Sociedad Médica de Santiago, en sus diversas categorías, indicándose el monto de las cuotas anuales que cada uno paga a la Sociedad. Ello permitiría aclarar si dichas cuotas cubren el costo de producción de la Revista, que reciben como uno de los beneficios de su membresía.

$2^{\circ}$ Conocer la evolución en los últimos años del número de socios que pagan cuotas permitiría estimar el efecto que ha tenido su versión electrónica, disponible en "open access", y predecir el futuro del sistema actual de "pago por los suscriptores".

$3^{\circ}$ Actualmente, en la Revista Médica de Chile la proporción de autores que reconocen haber recibido apoyo financiero institucional (Fondecyt $\mathrm{u}$ otros) es inferior al 30\% de los manuscritos publicados. Por ende, 70\% de los autores deberían pagar cualquier cobro de su propio peculio.

Un eventual cambio en la política de financiamiento de la Revista Médica de Chile debería realizarse con la máxima transparencia, como una decisión adecuadamente informada y aprobada por el Directorio de la Sociedad Médica de Santiago, propietaria de la Revista. A los autores de manuscritos se les debe advertir que el manejo editorial de sus manuscritos y las decisiones de los editores sobre aceptarlos o rechazarlos, así como la cronología del proceso hasta la publicación del manuscrito, ocurrirán absolutamente al margen de su capacidad de pagar cualquier cobro exigido por la Sociedad Médica de Santiago.

Esta es una oportunidad para que los lectores de la Revista y los autores de manuscritos contribuyan con opiniones, mediante Cartas al Editor.

\section{Referencias}

1. House of Commons Science and Technology Committee. Scientific publications: Free for all? London: Stationery Office, 2004 [Disponible en: www.publications.parliament.uk/pa/cm200304/cmselect/cmsctech/399/399.pdf ; consultado el 23 de enero de 2006 y el 28 de diciembre de 2011].

2. Lane Medical Library, Stanford University. Publishing Models. [Disponible en: http://lane.stanford.edu/help/ openaccess/models.html; consultado el 28 de diciembre de 2011].

3. Schroter S, Tite L. Open access publishing and authorpays business models: a survey of authors' knowledge and perceptions. J R Soc Med 2006; 99: 141-8.

4. Ranasinghe P, Perera S, Abeygunasekara AM. The process and costs of publishing medical journals in Sri Lanka: an economic evaluation. BMJ Open 2011. [Disponible en http://bmjopen.bmj.com; consultado el $16 \mathrm{de}$ diciembre de 2011].

5. King DW. An approach to Open Access Author Payment. D-Lib Magazine 2010; 16 (3/4). [Disponible en: http:// www.dlib.org/dlib/march10/king/03king.print.html; consultado el 28 de diciembre de 2011].

6. Frantsväg JE. The role of advertising in financing open access journals. First Monday 2010; 15 (3). [Disponible en http://firstmonday.org/htbin/cgiwrap/bin/ojs/index. $\mathrm{php/fm/article/viewArticle/2777/2478;} \mathrm{consultado} \mathrm{el} 16$ de diciembre de 2011].

7. Gores GJ. Open Access Journals: Why Are We Not There Yet? (Comments from the Editors). HEPATOLOGY 2010; 52 (6): 1869-71.

8. Open Journal of Internal Medicine. Scientific Research Publishing [Disponible en www.scirp.org; consultado el 6 de enero de 2012].

9. PLoS One [Disponible en http://www.plosone.org; consultado el 5 de enero de 2012].

10. Larraín C. La Sociedad Médica de Santiago y el Desarrollo Histórico de la Medicina en Chile. Imprenta Salesianos, Santiago de Chile, 2002. 